\title{
Pulling out Threads from the Cosmic Tapestry: Defining Filaments of Galaxies
}

\author{
Kevin A. Pimbblet ${ }^{\mathrm{A}, \mathrm{B}}$ \\ A Department of Physics, University of Queensland, Brisbane, QLD 4072, Australia \\ B E-mail: pimbblet@physics.uq.edu.au
}

\begin{abstract}
Filaments of galaxies are the dominant feature of modern large scale redshift surveys. They can account for up to perhaps half of the baryonic mass budget of the Universe and their distribution and abundance can help constrain cosmological models. However, there remains no single, definitive way in which to detect, describe and define what filaments are and their extent. This work examines a number of physically motivated, as well as statistical, methods that can be used to define filaments and examines their relative merits.
\end{abstract}

Keywords: large scale structure of Universe - cosmology: observations - methods: observational

\section{Motivation}

What is a filament of galaxies (FOG)? Although at first glance, this is a seemingly innocuous, benign and near-trivial question, there is not really a straight forward answer to it. Many authors, including the present one, have recently been searching for more concrete definitions and hence, also, methods of finding and detecting FOGs (e.g. Pimbblet 2005 and references therein).

The present work is therefore a timely review of the (growing) myriad of approaches that exist to define and detect FOGs in an attempt to answer the question of what a FOG actually is. In a lot of ways, investigations of FOGs nowadays are arguably analogous to where the investigations of galaxy clusters stood at about one half of a century ago (see Abell 1965 for an excellent, albeit somewhat dated by todays standards, review of galaxy clustering). Undoubtedly, the reason for the recent flurry of activity into investigating and characterizing FOGs has to be the availability of modern, high-quality and, most importantly, wide-field redshift surveys such as the 2dF Galaxy Redshift Survey (2dFGRS; e.g. Colless et al. 2001), the Sloan Digital Sky Survey (SDSS; e.g. Abazajian et al. 2004), the 6dF Galaxy Survey (6dFGS; e.g. Jones et al. 2004) and the Las Campanas Redshift Survey (LCRS; e.g. Shectman et al. 1996).

Having written that, the discovery of significant segments of large-scale structure other than galaxy clusters - sheets, filaments and walls of galaxies - is not a new thing. Geller \& Huchra (1989) famously cartographed the 'Great Wall' from the CfA redshift survey (e.g. Huchra et al. 1983): a highly significant feature that stretches for at least $170 \times 60 h_{100}^{-1} \mathrm{Mpc}$ at $c z \approx 7500 \mathrm{~km} \mathrm{~s}^{-1}$ (Figure 1). So significant is this detection, that it should even have an imprint on the cosmic microwave background radiation (Atrio-Barandela \& Kashlinsky 1992; see also Chodorowski 1994).

What else may we expect from FOGs? We know that in hierarchical structure formation modeling there has long been the prediction that galaxy clusters grow through repeated mergers with other galaxy clusters (and galaxy groups) together with continuous accretion of their surrounding matter (e.g. Zeldovich, Einasto \& Shandarin 1982; Katz et al. 1996; Jenkins et al. 1998; Colberg et al. 2000; see also Bond, Kofman \& Pogosyan 1996). We also know that the accretion process occurs in a highly non-isotropic manner: galaxy filaments funnel matter onto large clusters along preferred directions 


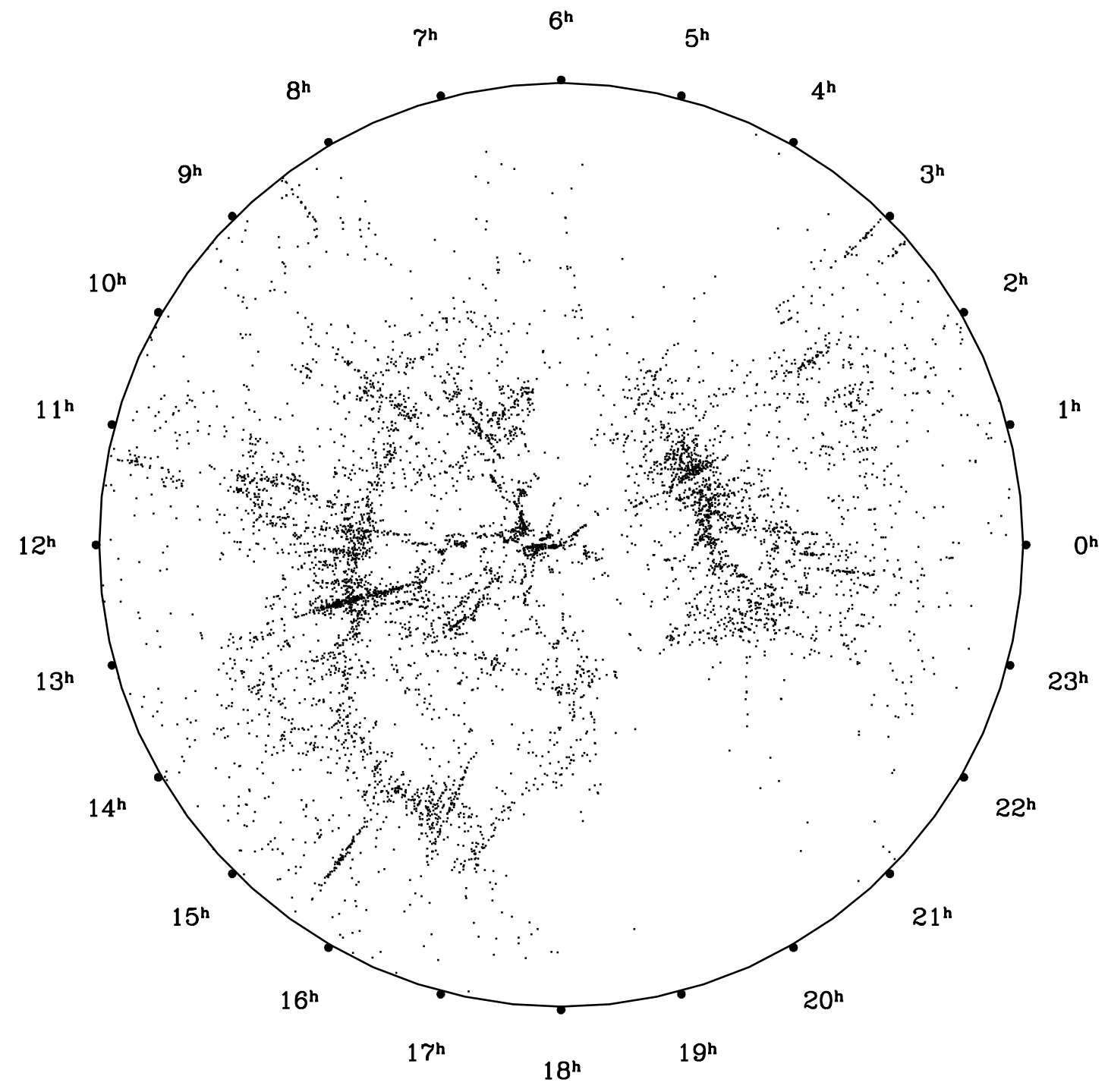

Figure 1: The Great Wall of Geller \& Huchra (1989) reconstructed using J2000 coordinates from the November 1993 public data release of the CfA dataset. Following Geller \& Huchra (1989), all galaxies within a declination range of $20<\delta<40$ are plotted with no cut in magnitude made. The radius of the circle is $15000 \mathrm{~km} \mathrm{~s}^{-1}$. The Great Wall can be seen extending outward from 13 hours. 
(see Pimbblet 2005; Ebeling, Barrett \& Donovan 2004; Kodama et al. 2001). Beyond a few virial radii from galaxy clusters centres, FOGs are predicted to weave a complex, web- or sponge-like tapestry that gives surveys like SDSS, 6dFGS, 2dFGRS \& LCRS their distinctive appearance (Figure 2 see also the 2dFGRS homepage at www.mso.anu.edu.au/2dFGRS ).

We also know that FOGs are highly important for the mass budget of the Universe (e.g. Colberg et al. 1999). Indeed, Cen \& Ostriker (1999) show that for a $\Lambda$ cold dark matter $(\Lambda C D M)$ Universe, a large fraction, perhaps as much as half (Fukugita, Hogan \& Peebles 1998), of baryonic material will not have been observed as it is situated in the inter-cluster media in a hot and tenuous gaseous phase. Along with the dark matter component and perhaps up to a quarter of the galaxian population, these baryons are preferentially situated in (inter-cluster) FOGs. Moreover, FOGs can provide tests of structure formation (c.f. Colberg, Krughoff \& Connolly 2004 with Pimbblet, Drinkwater \& Hawkrigg 2004) and cluster evolution (see Colberg et al. 1999). They can also be useful in ascertaining the homogeneity scale of our Universe (if, indeed one considers there to be a homogeneity scale; e.g. Coleman \& Pietronero 1992 and references therein). Certainly, given that objects with scale lengths $>150 h_{100}^{-1} \mathrm{Mpc}$ exist and are not chance superpositions, we should be questioning the validity of the cosmological assumption up to such lengths and beyond.

The rest of this paper plans out as follows. In Section 2, we investigate the numerous methods that one can employ to detect FOGs and explore their relative merits. In Section 3 we discuss the findings and present our conclusions.

\section{Detection}

Already we have seen a number of properties of FOGs. If sufficiently large, then they can cause a decrement in the cosmic microwave background radiation. They also possess multi-wavelength visibility (visual; X-ray; etc.). We describe below how one may take advantage of such properties to detect them in a given dataset.

\subsection{Optical Overdensity}

At a very simple level, a FOG is merely an overdensity of galaxies compared to the local field ${ }^{1}$ (or void) level. Pimbblet \& Drinkwater (2004) use this fact to find a relatively short $\left(\sim 6 h_{100}^{-1} \mathrm{Mpc}\right)$ filament between the two close (both in redshift and spatially) galaxy clusters ACO1079 and ACO1084. Mathematically, one can readily compute this galaxy excess as

$$
N_{\text {filament }}=N_{\text {filament }+ \text { field }}-N_{\text {field }}
$$

Should the observed field sample be too close to the observed filament sample it will obviously contain some (small but non-negligible) amount of contamination:

$$
N_{\text {field }}^{\prime}=N_{\text {field }}+\gamma N_{\text {filament }}
$$

where $\gamma$ is the ratio between the galaxy densities of the filament and field populations (Paolillo et al. 2001). Substituting $N_{\text {field }}^{\prime}$ instead of $N_{\text {field }}$ from Eq. (2) into Eq. (1) gives

$$
N_{\text {filament }}^{\prime}=N_{\text {filament }}(1-\gamma)
$$

In Figure 3 we plot an adaptation of the result obtained by Pimbblet \& Drinkwater (2004) utilizing this particular method. Most of the excess galaxy population is faint, with only a few brighter members. Moreover, only a small fraction $(\approx 30$ per cent) of these galaxies have colours consistent with earlytype galaxies from the two clusters colour-magnitude relations (Pimbblet et al. 2002). In shallow (perhaps, mono-colour) surveys with no supporting redshift information, therefore, such an approach is probably not very efficient nor exceedingly sensitive and may be somewhat prone to large errors.

\footnotetext{
${ }^{1}$ Here, we use the term 'field' to mean the 'average background' level.
} 


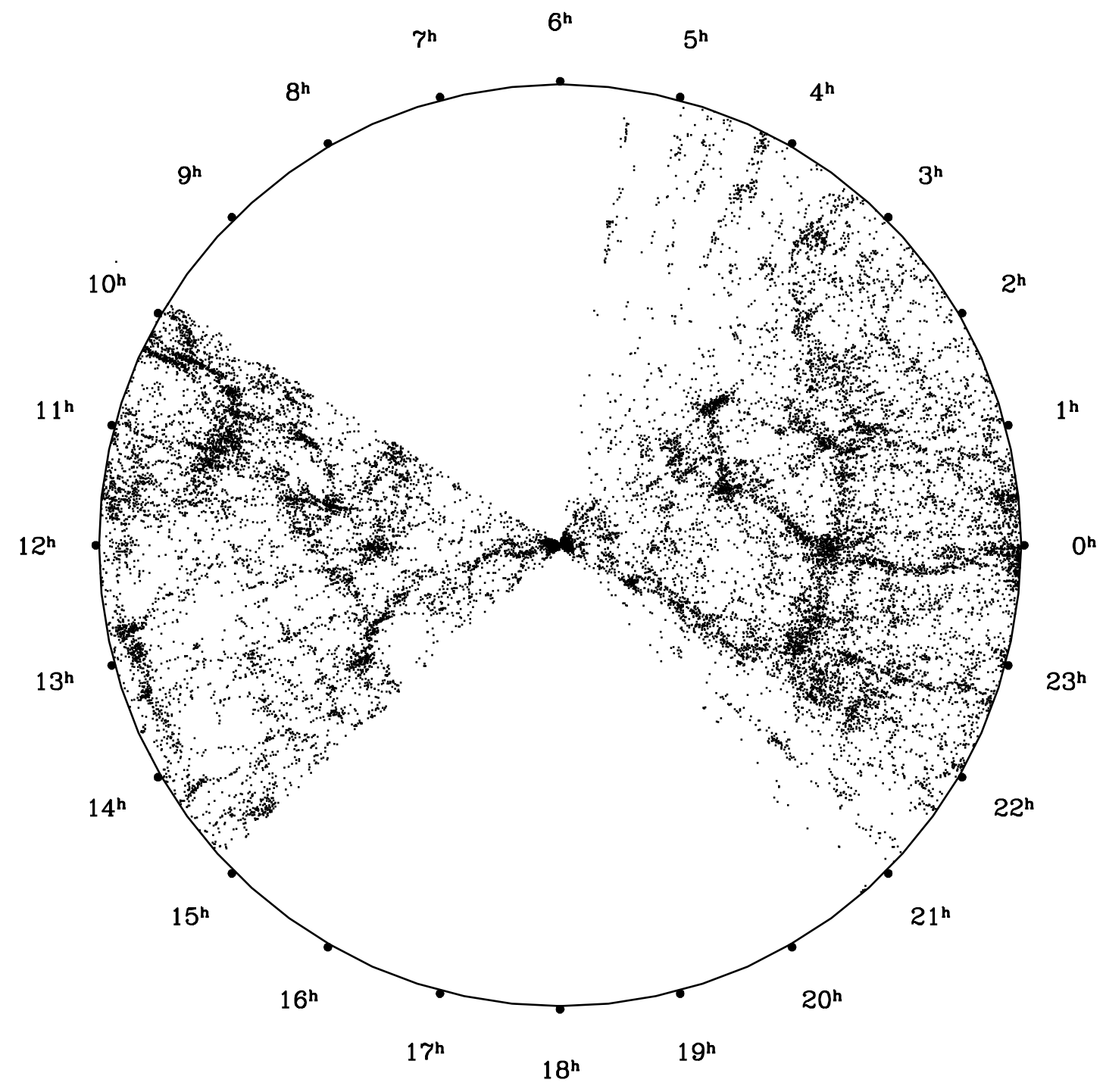

Figure 2: As for Figure 1 but using data from 2dFGRS. No cut has been made in declination or $b_{J}$ magnitude, although the plot has been constrained to the same redshift limit as Figure 1 albeit with a different declination range. Note the complex manner in which FOGs weave through the structure and its qualitative similarity to the CfA survey. 


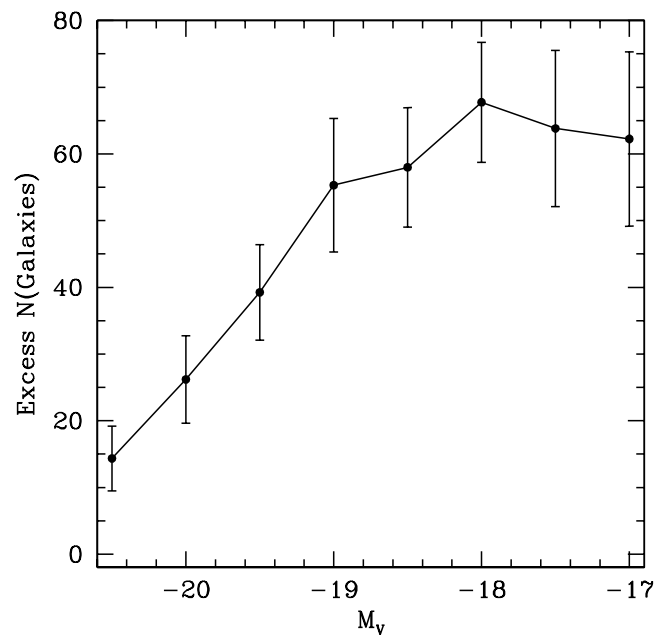

Figure 3: Number of galaxies in excess of the field population (i.e. the filament population) as a function of absolute magnitude (adapted from the investigation of Pimbblet \& Drinkwater 2004). The $\pm 1 \sigma$ errorbars come from a consideration of Poissonian errors and the variance galaxy number density. Whilst there are only a few brighter galaxies, the FOG contains many more fainter ones.

\section{$2.2 \quad$ X-ray}

Given that a non-negligible fraction of baryonic material in a $\Lambda$ CDM Universe may exist as hot intercluster gas, one can also consider looking for FOGs in X-ray band passes. Using the ROSAT All-Sky Survey data, Briel \& Henry (1995) attempted just this by combining together the inter-cluster regions of 40 cluster pairs. Although they failed to find any X-ray emitting FOG, they did place an upper limit on the X-ray surface brightness of $4 \times 10^{-16} \mathrm{ergs} \mathrm{cm}^{-2} \mathrm{~s}^{-1}(0.5-2.0 \mathrm{keV})$.

Scharf et al. (2000) make a $5 \sigma$ joint X-ray/optical detection of $>12 h_{50}^{-1} \mathrm{Mpc}(0.5 \mathrm{deg}) \mathrm{FOG}$ with a surface brightness of $1.6 \times 10^{-16} \mathrm{ergs} \mathrm{cm}^{-2} \mathrm{~s}^{-1}$. The count rate for this filament, however, is $2-3 \sigma$ above background levels.

In the Shapley supercluster meanwhile, Kull \& Böhringer (1999) find a promising extended X-ray emission between a close cluster pair that is $\sim 2.5$ times brighter than Briel \& Henry's (1995) bound. The only problem here is that Scharf et al. (2000) note that the X-ray emission could be ejecta due to the clusters interacting (merging) with one another rather than material falling in from an actual filament.

More promising progress on this front has been made by Tittley \& Henriksen (2001) and Durret et al. (2003). The former detect a gaseous FOG between ACO3391 and ACO3395 that has a minimum flux of $1.3 \times 10^{-12} \mathrm{ergs} \mathrm{cm}^{-2} \mathrm{~s}^{-1}(0.8-10 \mathrm{keV})$ and represents at least 2 per cent of the total mass of the system. The latter team study the ACO85 cluster complex and find a highly elongated filamentary structure. Again, however, this filament may the result of cluster interactions and not a true FOG in the large-scale structure sense.

It would seem that whilst one may expect there to be significant X-ray emission from baryonic material contained in FOGs, we are not quite detecting it with sufficient regularity or confidence to use X-ray emission as the primary tool for FOG detection (unlike in the case of galaxy clusters where X-ray detections are made with much more confidence; e.g. Ebeling et al. 1996).

\subsection{Lensing}

Pogosyan et al. (1998) point out that FOGs that connect together neighbouring galaxy clusters should have sufficient surface mass density as to be detectable in the weak lensing regime. Indeed, weak lensing would only depend upon the projected density and not the square of the projected density like X-rays are (Pogosyan et al. 1998) and therefore it may constitute an altogether better way of 
detecting and defining FOGs. There are a small number of investigations that have been proceeding in this direction.

Kaiser et al. (1998) perform a lensing analysis on the $z=0.4$ supercluster MS $0302+17$ and find FOG between two of its three component galaxy clusters. The detection has remained dubious, however, as there may be foreground structure interfering, perhaps some edge of chip effects and residual systematics in the point spread function anisotropy correction involved (Gavazzi et al. 2004). Indeed, Gavazzi et al. (2004) report that they cannot independently confirm the detection of this particular FOG.

Meanwhile, in other investigations, Clowe et al. (1998) report on the detection of a FOG extending from the $z=0.8$ rich cluster RXJ $1716+67$. However, the size of their imaging is small and it is thus unknown how far this filament extends in the direction of a nearby cluster. Gray et al. (2002) examines the ACO901/902 supercluster and find a FOG present. The significance of the detection is, however, small. Superposed with this is the issue that the filament lies in the inter-chip region of the analysis. Nonetheless, this remains a relatively good detection when compared to the problems that Kaiser et al. (1998) encounter.

Yet to date, arguably one of the best weak lensing FOG detections has to be that of Dietrich et al. (2004) between ACO222 and ACO223. Not only is the filament detected by weak lensing, but Dietrich et al. (2004) supply supporting evidence from X-ray emission and increased galaxy density between the clusters. Dietrich et al. (2004) point out, however, that they could not find an objective way to define their filament and in this respect their filament candidate is not very different to that of Kaiser et al. (1998).

So, it would appear that given imaging of sufficient quality and depth, weak lensing could provide an excellent way of detecting FOGs, most especially in combination with other methods (e.g. X-ray; see above).

\subsection{Redshifts}

Redshifts of regions around galaxy clusters can provide concrete determinations of the presence of FOGs. For example, Ebeling, Barrett \& Donovan (2004) report on a $4 h_{70}^{-1} \mathrm{Mpc}$ filament that is feeding the growth of the massive cluster MACS J0717.5+3745 at $z=0.55$. Its extent beyond the virial radius of the cluster means that it cannot be the remnant of some previous interaction or merger whilst its colours are quite consistent with the colour-magnitude relation (CMR; e.g. Visvanathan \& Sandage 1977; Bower, Lucey \& Ellis 1992) Indeed, the CMR and other photometric redshift techniques can also help to better define FOGs. Kodama et al. (2001) report several 'octopus'-like tentacles around ACO851 $(z=0.41)$ which have colours entirely consistent with the CMR of the cluster itself. Pimbblet, Edge \& Couch (2005) locate a large scale wall covering at least $40 h_{100}^{-1} \mathrm{Mpc}$ situated in front of $\operatorname{ACO} 22(z=0.14$; Figure 4$)$. Not only does this wall exhibit a CMR similar to ACO22, but it also has a Butcher \& Oemler (1984; see Pimbblet 2003 for a review of the Butcher-Oemler effect) blue fraction that does not change significantly between the cluster and the wall (Figure 4).

The above are examples of FOGs around individual clusters. Of course, it is the modern large redshift surveys such as 2dFGRS, 6dFGS, SDSS and LCRS that are providing the community with an unprecedented view of the very large scale structure of the Universe (e.g. Figure 21). With such large datasets, finding individual filaments can become as easy as looking at the regions between two galaxy clusters in three dimensional space and making an appropriate cut at some galaxy density threshold ${ }^{2}$ to determine if there is a significant overdensity of galaxies present (e.g. Pimbblet, Drinkwater \& Hawkrigg 2004). One potential pitfall is that one may mistake a redshift space distortion (see Hawkins et al. 2003) for a FOG. Pimbblet, Drinkwater \& Hawkrigg (2004) circumvent this by only considering cluster-cluster pairs within $1000 \mathrm{kms}^{-1}$ of each other and check the FOG distribution angles along the line of sight to ensure that no 'fingers of god' are mistaken for FOGs (and conversely, no end-on FOG is mistaken for a cluster!). Also, surveys like 2dFGRS only cream off the very luminous galaxies (and even at bright magnitudes, are incomplete; Cross et al. 2004). They tell us little about the low

\footnotetext{
${ }^{2}$ Pimbblet, Edge \& Couch (2005) note that the typical surface density of FOGs is of the order 10 bright (brighter than say $\left.M^{*}+2\right)$ galaxies per square $\mathrm{h}^{-1} \mathrm{Mpc}$.
} 


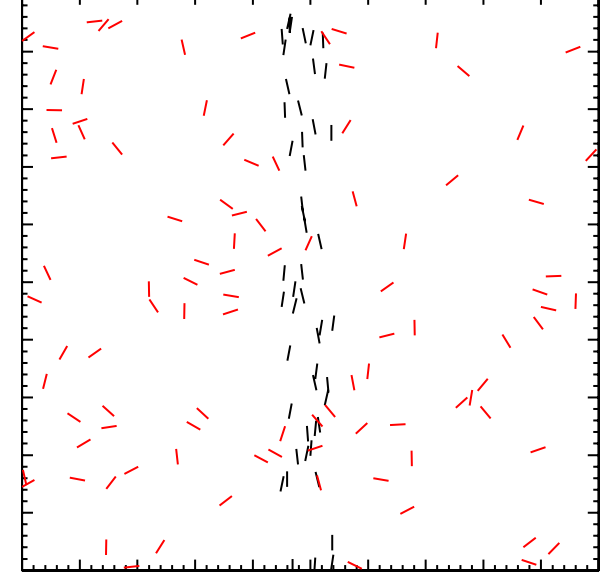

Figure 6: If one makes use of galaxy angles (i.e. vectors), then the problem posed by Figure 5 potentially becomes much easier to solve. Here, the members of the FOG have orientations of $\theta=90 \pm 15$ deg, whereas the interlopers (red) have purely random orientation angles.

incomplete by about 10 to 20 per cent at all magnitudes (Cross et al. 2004; Pimbblet et al. 2001) - a fact that makes MST potentially a poorer choice for analyzing large scale structure with than other methods.

Such other statistical methods, that here we will mention only briefly, include the use of Voronoi (and the complementary Delaunay) tessellations (e.g. van de Weygaert 1994). Essentially, the Voronoi tessellation can be thought of as constructing a skeleton of the Universe by simply finding the bisection line between a single point and every other point. This process is repeated for each point and then the Voronoi tessellation is then the unification of all the halfplanes that have been created (see van de Weygaert 1994 for more indepth detail). Analysis of the cell-like structures of the Voronoi skeleton can inform one about the underlying galaxy distribution, although direct detection of FOGs from these tessellations remains a relatively unattempted task. Minkowski functionals such as SHAPEFINDERS (e.g. Bharadwaj et al. 2000; Pandey \& Bharadwaj 2005; see also Shandarin, Sheth \& Sahni 2004) and the genus statistics (e.g. Hoyle et al. 2002; Hoyle, Vogeley \& Gott 2002) can also provide us with a direct way of analyzing the structure of the galaxy distribution. Moreover, they can also provide a direct measure of the 'filamentarity' and 'planarity' of the Universe (e.g. Schmalzing et al. 1999) and one can readily delineate FOGs from them by using an isodensity contour cut. Finally, we should also mention that there are a host of other marked point processes (Stoica et al. 2005 and references therein) which are also capable of recovering individual FOGs. All of these methods highlight the presence of FOGs within redshift surveys to varying degrees.

\section{Discussion and Conclusions}

Given the above methods to detect FOGs, there is a large amount of literature dedicated to their dissemination. One issue that seems to be prominent in the literature is the mixed nomenclature for FOGs. Many authors refer to them as 'walls' (Geller \& Huchra 1989), others call them 'filaments', some use the term 'sheets' or 'pancakes'. So what is the difference between all these terms? Pimbblet, Drinkwater \& Hawkrigg (2004) and Colberg, Krughoff \& Connolly (2004) attempt to refine these definitions by dividing detected FOGs into several categories based upon their visual morphology. So the difference between a filament and a wall then becomes a matter of how thick (or, equally, how wide) the FOG is in three-dimensional space (i.e. a filament will have depth $\approx$ width). Sheets are then synonymous with walls. Is this kind of morphological classification useful? Given that walls appear to be much, much rarer than 'normal' filaments (Pimbblet, Drinkwater \& Hawkrigg 2004) and unlike filaments, they do not possess non-isotropic galaxy orientations (Pimbblet 2005) - yes. Their relative abundances (also filling factors) and lengths should help us to better constrain the ideal cosmological paradigm (e.g. in $\Lambda$ cold dark matter cosmologies, studies of FOGs can readily exclude bias parameters of $b>1.5$; Bharadwaj \& Pandey 2004) as can the number of FOGs connected to clusters of a given 
mass (Colberg, Krughoff \& Connolly 2004).

At the outset of this work, the question 'what is a filament of galaxies?' was posed. This work has reviewed a number of methods for finding, detecting and defining FOGs in datasets of varying complexity. Those that are more physically motivated (gravitational weak lensing searches; X-ray searches) appear to be an optimal way to detecting them (especially in unison), but yet, they remain a time-intensive method owing to the required amount of observing time to get down to sufficient limiting magnitudes and fluxes.

We have also investigated how FOGs are detected in large redshift surveys using a variety of methods ranging from simple isodensity thresholding to more involved statistics like the MST. Here, it seems that even the very simple approaches can yield useful results, such as the distribution and abundances of FOG lengths, that are in remarkable agreement with theory.

\section{Acknowledgments}

KAP thanks Michael Drinkwater, Alastair Edge and Mary Hawkrigg for their support. This work is financed through the award of a University of Queensland EPSA Research Fellowship and a UQRSF grant.

On a final note, I also wish to express my sincere gratitude to the two referees, Dominique Proust and Tony Fairall, who provided quick and useful reports that have improved the quality of this work.

\section{References}

Abazajian K., et al., 2004, AJ, 128, 502

Abell G. O., 1965, ARA\&A, 3, 1

Arias-Castro E., Donoho D., Huo X., Tovey C., 2004, Advances in Applied Probability, submitted (www-stat.stanford.edu / donoho / Reports / 2004 / CTD-Arias-etal.pdf)

Atrio-Barandela F., Kashlinsky A., 1992, ApJ, 390, 322

Barrow J. D., Bhavsar S. P., Sonoda D. H., 1985, MNRAS, 216, 17

Bharadwaj S., Pandey B., 2004, ApJ, 615, 1

Bharadwaj S., Sahni V., Sathyaprakash B. S., Shandarin S. F., Yess C., 2000, ApJ, 528, 21

Bhavsar S. P., Ling E. N., 1988, ApJ, 331, L63

Binggeli B., 1982, A\&A, 107, 338

Bond J. R., Kofman L., Pogosyan, D., 1996, Nature, 380, 603

Bower R. G., Lucey J. R., Ellis R. S., 1992, MNRAS, 254, 601

Briel U. G., Henry J. P., 1995, A\&A, 302, L9

Butcher, H. \& Oemler, A. 1984, ApJ, 285, 426

Cen R., Ostriker J. P., 1999, ApJ, 514, 1

Chodorowski M., 1994, MNRAS, 266, 897

Clowe D., Luppino G. A., Kaiser N., Henry J. P., Gioia I. M., 1998, ApJ, 497, L61

Colberg J. M., Krughoff K. S., Connolly A. J., 2004, MNRAS in press (astro-ph/0406665)

Colberg J. M., et al., 2000, MNRAS, 319, 209 
Colberg J. M., White S. D. M., Jenkins A., Pearce F. R., 1999, MNRAS, 308, 593

Coleman P. H., Pietronero L., 1992, PhR, 213, 311

Cross N. J. G., Driver S. P., Liske J., Lemon D. J., Peacock J. A., Cole S., Norberg P., Sutherland W. J., 2004, MNRAS, 349, 576

Dietrich J. P., Schneider P., Clowe D., Romano-Diaz E., Kerp J., 2004, preprint, astro-ph/0406541

Doroshkevich A. G., Fong R., McCracken H. J., Ratcliffe A., Shanks T., Turchaninov V. I., 2000, MNRAS, 315, 767

Doroshkevich A., Tucker D. L., Allam S., Way M. J., 2004, A\&A, 418, 7

Durret F., Lima Neto G. B., Forman W., Churazov E., 2003, A\&A, 403, L29

Ebeling H., Barrett E., Donovan D., 2004, ApJ, 609, L49

Ebeling H., Voges W., Bohringer H., Edge A. C., Huchra J. P., Briel U. G., 1996, MNRAS, 281, 799

Fairall A., Turner D., Pretorius M. L., Wiehahn M., McBride V., de Vaux G., Woudt P. A., 2004, preprint, astro-ph/0411437

Fukugita M., Hogan C. J., Peebles P. J. E., 1998, ApJ, 503, 518

Gavazzi R., Mellier Y., Fort B., Cuillandre J.-C., Dantel-Fort M., 2004, A\&A, 422, 407

Geller M. J., Huchra J. P., 1989, Sci, 246, 897

Gray M. E., Taylor A. N., Meisenheimer K., Dye S., Wolf C., Thommes E., 2002, ApJ, 568, 141

Hawkins E., et al., 2003, MNRAS, 346, 78

Hoyle F., et al., 2002, ApJ, 580, 663

Hoyle F., Vogeley M. S., Gott J. R. I., 2002, ApJ, 570, 44

Huchra, J., Davis, M., Latham, D., \& Tonry, J. 1983, ApJS, 52, 89

Jenkins A., et al., 1998, ApJ, 499, 20

Jones D. H., et al., 2004, MNRAS, 355, 747

Kaiser N., Wilson G., Luppino G., Kofman L., Gioia I., Metzger M., Dahle H., 1998, preprint, astro-ph/9809268

Katz N., Weinberg D. H., Hernquist L., Miralda-Escude J., 1996, ApJ, 457, L57

Kodama T., Smail I., Nakata F., Okamura S., Bower R. G., 2001, ApJ, 562, L9

Krzewina L. G., Saslaw W. C., 1996, MNRAS, 278, 869

Kull A., Böhringer H., 1999, A\&A, 341, 23

Pandey B., Bharadwaj S., 2005, MNRAS, 357, 1068

Paolillo M., Andreon S., Longo G., Puddu E., Gal R. R., Scaramella R., Djorgovski S. G., de Carvalho R., 2001, A\&A, 367, 59

Pimbblet K. A., 2005, MNRAS, 358, 256

Pimbblet K. A., Edge A. C., Couch W. J., 2005, MNRAS, 357, L45

Pimbblet K. A., Bulmer M., 2005, PASA, 22, 1 
Pimbblet K. A., Drinkwater M. J., Hawkrigg M. C., 2004, MNRAS, 354, L61

Pimbblet K. A., Drinkwater M. J., 2004, MNRAS, 347, 137

Pimbblet K. A., 2003, PASA, 20, 294

Pimbblet K. A., Smail I., Kodama T., Couch W. J., Edge A. C., Zabludoff A. I., O'Hely E., 2002, MNRAS, 331, 333

Pimbblet K. A., Smail I., Edge A. C., Couch W. J., O'Hely E., Zabludoff A. I., 2001, MNRAS, 327, 588

Pogosyan D., Bond J. R., Kofman L., Wadsley J., 1998, in Wide Field Surveys in Cosmology, 14th IAP meeting held May 26-30, 1998 (Paris: Editions Frontieres), 61

Scharf C., Donahue M., Voit G. M., Rosati P., Postman M., 2000, ApJ, 528, L73

Schmalzing J., Buchert T., Melott A. L., Sahni V., Sathyaprakash B. S., Shandarin S. F., 1999, ApJ, 526,568

Shandarin S. F., Sheth J. V., Sahni V., 2004, MNRAS, 353, 162

Shectman S. A., Landy S. D., Oemler A., Tucker D. L., Lin H., Kirshner R. P., Schechter P. L., 1996, ApJ, 470, 172

Stoica R. S., Martinez V. J., Mateu J., Saar E., 2005, A\&A, in press astro-ph/0405370

Tittley E. R., Henriksen M., 2001, ApJ, 563, 673

van de Weygaert R., 1994, A\&A, 283, 361

Visvanathan N., Sandage A., 1977, ApJ, 216, 214

Zeldovich I. B., Einasto J., Shandarin S. F., 1982, Nature, 300, 407 